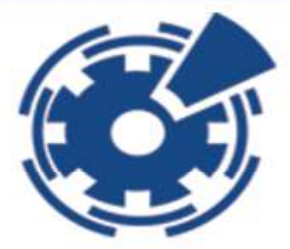

\title{
Characterization of Phenolic Compounds in Peel, Pulp and Seed Fractions of Zizyphus Jujube Fruit by UFLC-PDA and Determination of Antioxidant Activity
}

\author{
Aysun Yücetepe ${ }^{1 *}$, Ayhan Duran², Beraat Özçelik $^{3}$ \\ ${ }^{1,2}$ Aksaray University Engineering Faculty Food Engineering Department Tr68100 Aksaray/Turkey \\ ${ }^{3}$ Deparment of Food Engineering, Faculty of Chemical \& Metallurgical Engineering, \\ Istanbul Technical University, Tr34469, Istanbul/ Turkey
}

\begin{abstract}
The objective of this present study was to characterize peel, pulp and seed tissues of Zizyphus jujube, commonly known as "Jujube», to determine the total phenolic content, anthocyanin content, antioxidant activity and phenolic profile. Antioxidant potential of samples were measured with two different methods 2,2-diphenyl-2-picrylhydrazyl (DPPH) radical scavenging activity and cupric reducing capacity (CUPRAC). Monomeric anthocyanin contents of the samples were determined using the $\mathrm{pH}$ differential method. The total phenolic content of the extracts was determined according to the Folin Ciocalteu method. Moreover, an ultra-fast liquid chromatograph (UFLC) method with photodiode array detector (PDA) was used to identify total phenolic acids (gallic acid, 3,4dihydroxybenzoic acid, catechin, chlorogenic acid, caffeinne, 4phydroxybenzoic acid, epicatechin, epigallocatechingallate, caffeic acid, vanilic acid, syringic acid, rutin, p-coumaric acid, vanilin, sinapic acid, ferulic acid, rosemarinic acid, phloridzin, myricetin, ethyl 3,4-dihydroxybenzoate, luteolin, quercetin, trans-cinnamic acid, naringenin, apigenin, kaempferol, hesperetin, Isorhamnetin, rhamnetin, ladanein, pectolinaringenin, pinostrobin) in peel, pulp and seed phenolic extracts of jujube. Total phenolic content of peel, pulp and seed were determined as $27.16 \pm 155,33.74 \pm 060$ and $10.37 \pm 086 \mathrm{mg}$ gallic acid equivalent (GAE)/g dry weight, respectively. Antioxidant activities evaluated by DPPH were as follows $62.32 \pm 079$, $122.63 \pm 092$ and $15.52 \pm 080 \mathrm{mg}$ Trolox equivalent (TE)/g dry weight, respectively. Antioxidant activities evaluated by CUPRAC were as follows $60.62 \pm 1.92,69.91 \pm 0.92$ and $21.03 \pm 1.73 \mathrm{mg}$ Trolox (TE)/g dry weight, respectively. Anthocyanin contents of the samples found as $419.97 \pm 10.89,29.41 \pm 6.45$ and $24.05 \pm 5.18 \mathrm{mg}$ cyanidin 3-glucoside $100 \mathrm{~g}$ dry weight for peel, pulp and seed fractions, respectively. The results of UFLC analysis indicate that catechin and vanilic acid were the main phenolics in all fractions of jujube.
\end{abstract}

Keywords: Antioxidant activity; jujube; phenolics; Zizyphus jujube. 